\title{
Hallazgos radiológicos en un niño con espondiloencondrodisplasia
}

\section{Radiological findings of a boy with spondyloenchondrodysplasia}

\author{
Andressa Fiori Bortoli ${ }^{1}$ Vinícius de Borba Capaverde ${ }^{1}$ Fernanda Scalco Acco ${ }^{1}$ Andrea Kiss ${ }^{2}$ \\ José Antônio Monteiro Flores ${ }^{3}$ Rafael Fabiano Machado Rosa ${ }^{4,5}$
}

1 Universidad Federal de Ciencias de la Salud de Porto Alegre, Rio Grande do Sul, Brasil

${ }^{2}$ Genética Clínica, Santa Casa de Misericordia de Porto Alegre, Rio Grande do Sul, Brasil

${ }^{3}$ Servicio de Radiología Pediátrica, Hospital Pediátrico de Santo Antônio, Rio Grande do Sul, Brasil

${ }^{4}$ Genética Clínica, Universidad Federal de Ciencias de la Salud de Porto Alegre y Santa Casa de Misericordia de Porto Alegre, Rio Grande do Sul, Brasil

${ }^{5}$ Programa de Posgrado en Patología, Universidad Federal de Ciencias de la Salud de Porto Alegre, Rio Grande do Sul, Brasil

Rev Argent Radiol 2020;84:71-74.

Estimados editores,

Presentamos el caso de un niño con espondiloencondrodisplasia (SPENCD, por su sigla en inglés) haciendo hincapié en los hallazgos radiológicos. Se trata de un niño de 9 años de edad, de raza blanca, único hijo de progenitores jóvenes, sanos y no consanguíneos. El niño no tenía antecedentes familiares de enfermedades genéticas ni óseas. Nació a término por cesárea con un peso de $3.870 \mathrm{~g}$ $(\mathrm{DE}+0,63)$, una talla de $49 \mathrm{~cm}(\mathrm{DE}-0,44)$ y un puntaje de Apgar de 9 a los 5 minutos. Presentó infecciones recurrentes, incluyendo episodios de bronconeumonía, con varias internaciones desde el primer mes de vida. Se advirtió retraso del crecimiento a los 4 años de edad.

A los 8 años de edad, presentó fiebre recurrente de origen desconocido. En la evaluación clínica, a los 9 años de edad, el niño tenía un peso de $25,55 \mathrm{~kg}$ (DE-0,86), una talla de $120 \mathrm{~cm}$ $(D E-2,4)$ y un perímetro cefálico de $53,5 \mathrm{~cm}(\mathrm{DE}+0,72)$, pliegues epicánticos bilaterales, puente nasal bajo, narinas en anteroversión, orejas de implantación baja, leve desproporción entre los segmentos superior e inferior del cuerpo y hepatoesplenomegalia ( $\mathbf{F i g . 1 A}$ y B).

Su desarrollo neuropsicomotor e inteligencia se encontraban dentro del rango normal. Tampoco presentaba signos de déficit neurológico. Su dentadura era normal.

received

November 29, 2018

accepted

December 2, 2019
Address for correspondence Rafael Fabiano Machado Rosa, Rua Sarmento Leite, 245 / 403, Porto Alegre, Rio Grande do Sul, Brasil (e-mail: rfmrosa@gmail.com).
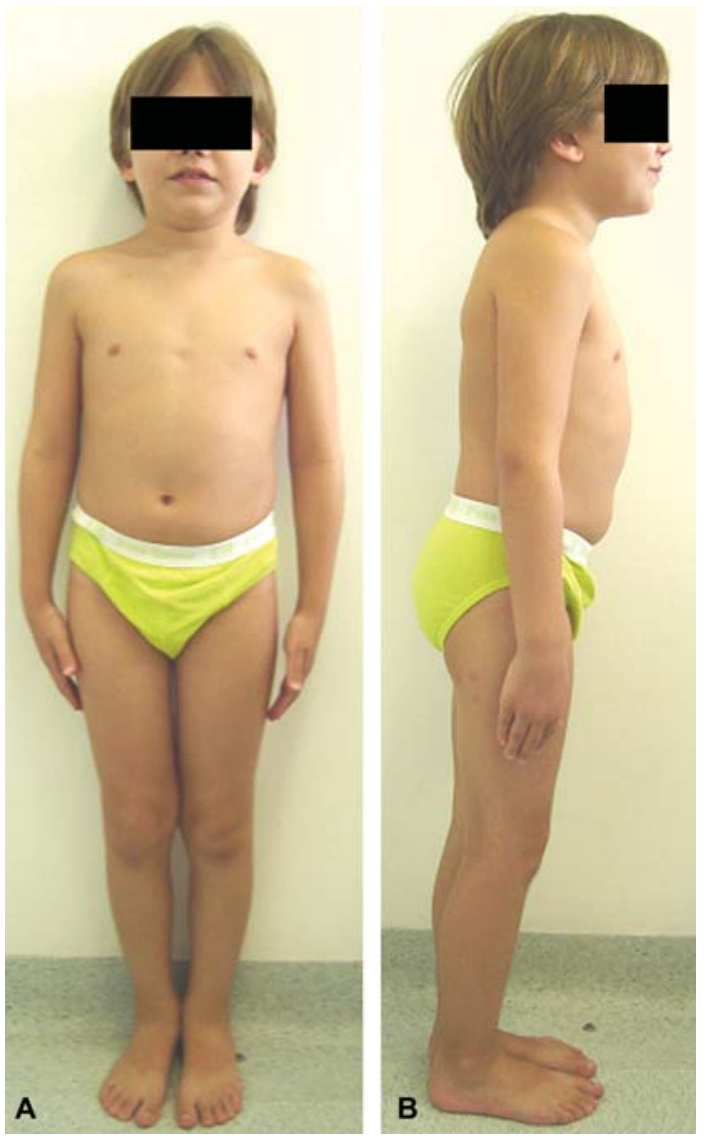

Fig. 1 Paciente de 9 años de edad con leve desproporción entre los segmentos superior e inferior del cuerpo (A y B).

Copyright @ $\odot$ 2020, Sociedad Argentina de Radiología. Publicado por Thieme Revinter Publicações Ltda., Rio de Janeiro, Brazil. Todos los derechos reservados.

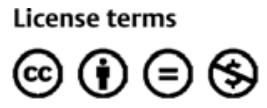

10.1055/s-0040-1701275 ISSN 1852-9992. 

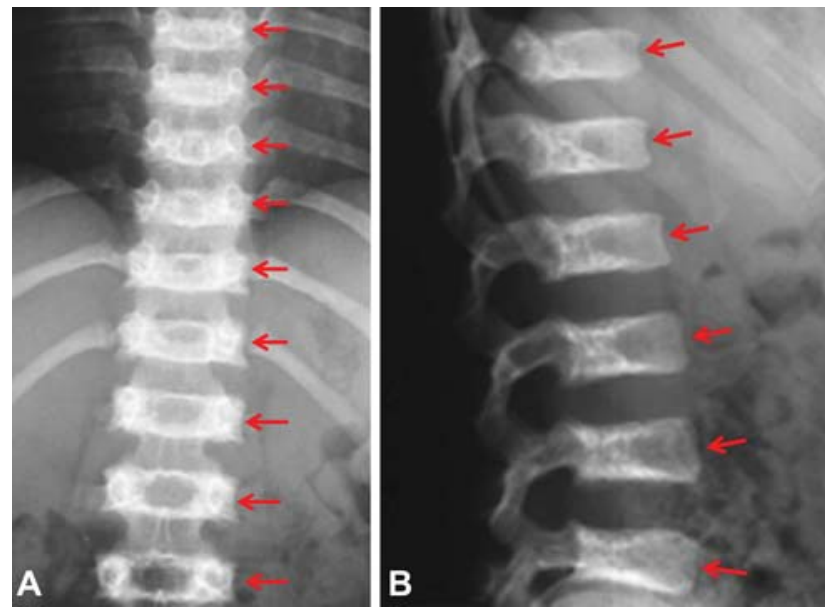

Fig. 2 Radiografías de columna que muestran la importante platispondilia (véanse las flechas rojas) y encondromas (A y B).

El examen radiológico de la columna verificó la presencia de vértebras con acentuada platiespondilia (importante reducción del diámetro vertical de las vértebras), hendiduras coronales y lesiones radiotransparentes (encondromas) que determinan irregularidad del platillo vertebral ( - Fig. 2A y B).

En los huesos largos, se observaban lesiones radiotransparentes que determinan irregularidad de las regiones metafisarias (3a y $3 \mathrm{~b}$ ) y deformidad de las epífisis de los fémures (3b y $3 c$ ), tibias (3b) y húmeros (3d y 3e) (-Fig. 3).

El niño presentaba retraso de la edad ósea. La ecografía abdominal confirmó la presencia de hepatoesplenomegalia. La evaluación inmunológica sugirió inmunodeficiencia combinada humoral y celular. Los resultados de la resonancia magnética de cerebro, análisis de cariotipo mediante bandeo GTG de alta resolución y análisis metabólico para la detección de enfermedades por depósito fueron normales.

A los 9 años de edad, el niño seguía presentando lesiones herpéticas recurrentes de difícil resolución. Un año más tarde, desarrolló insuficiencia renal y se le diagnosticó lupus eritematoso sistémico (LES), confirmado por biopsia renal. Luego, evolucionó con varias internaciones por artritis de rodilla a causa del LES. Sus padres presentaban fenotipo normal. El análisis de secuencias realizado según Lausch y col. ${ }^{1}$ mostró una mutación patogénica homocigótica en ACP5: c.791T > A (alteración de la proteína: p.Met264Lys). Ambos progenitores eran heterocigotos para esa mutación. Por lo tanto, la evaluación genética, a través del análisis de los hallazgos clínicos y los resultados de los estudios de laboratorio y de diagnóstico por imagen, fue compatible con el diagnóstico de SPENCD.

Los hallazgos radiológicos específicos observados en nuestro paciente, principalmente en las vértebras, la porción distal del radio y del cúbito y las rodillas, permitieron diagnosticar SPENCD. ${ }^{2,3}$ En una publicación, Schorr y col. ${ }^{4}$ plantearon el diagnóstico de SPENCD en base a los hallazgos óseos, que comprenden la presencia de platispondilia de grado variable, con irregularidad de los platillos vertebrales superior e inferior, presencia de lesiones nodulares localizadas inicialmente (o solo) en el tercio o la mitad posterior del cuerpo vertebral y lesiones no osificantes radiotransparentes, en sacabocados, redondeadas o multilobuladas que se extienden desde el cartílago de crecimiento a la metáfisis y diáfisis. Esas lesiones pueden ser de gravedad variable y aparecer solo en los huesos largos (típicamente en la zona distal de la rodilla, peroné proximal, radio distal y cúbito), pero también pueden observarse en otros sitios de crecimiento endocondral, como la cresta ilíaca. 4,5
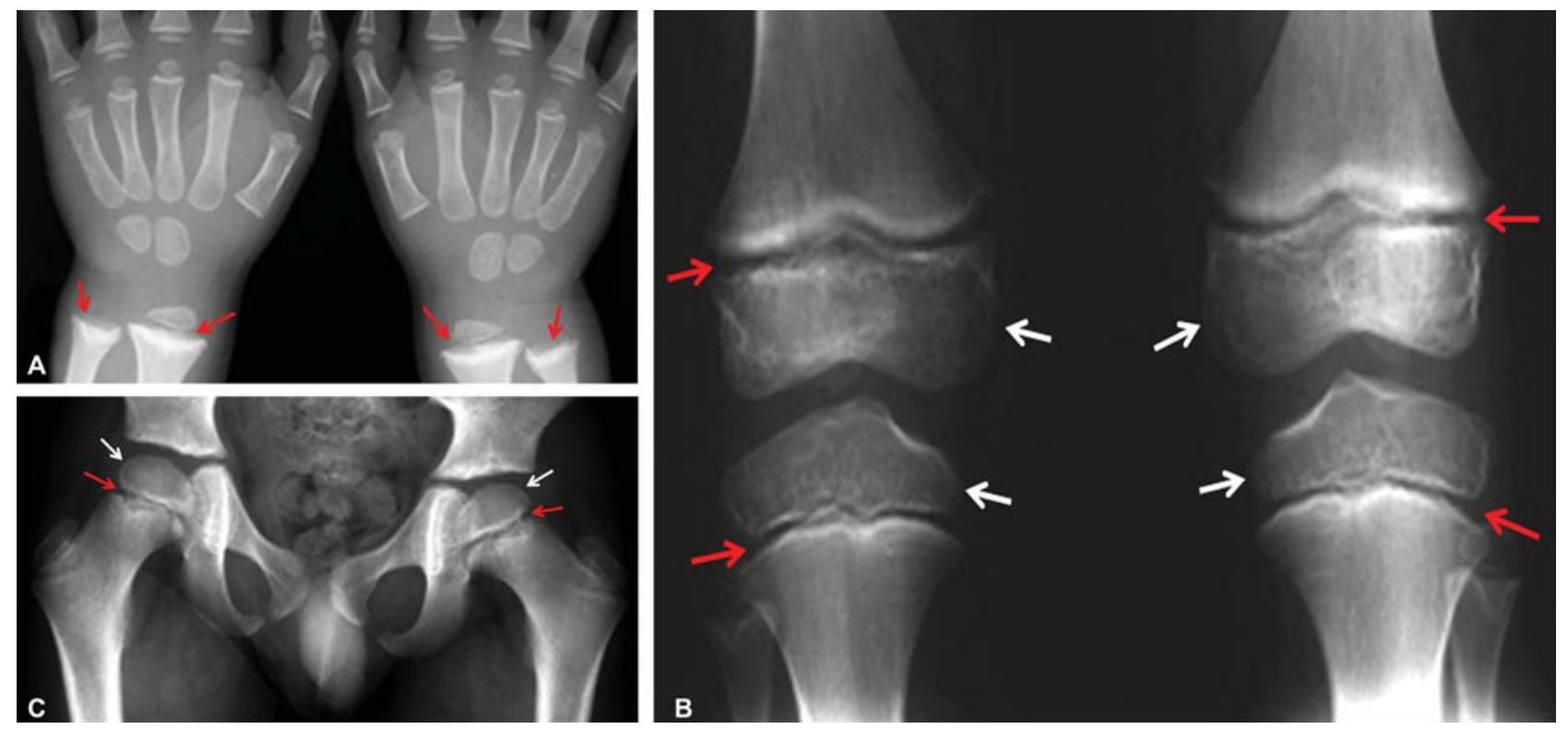

Fig. 3 Radiografías que muestran irregularidad de las metáfisis de los huesos largos (A-E) (flechas rojas) y deformidad de las epífisis de fémures (B y C), tibias (B) y húmeros (D y E) (flechas blancas). 

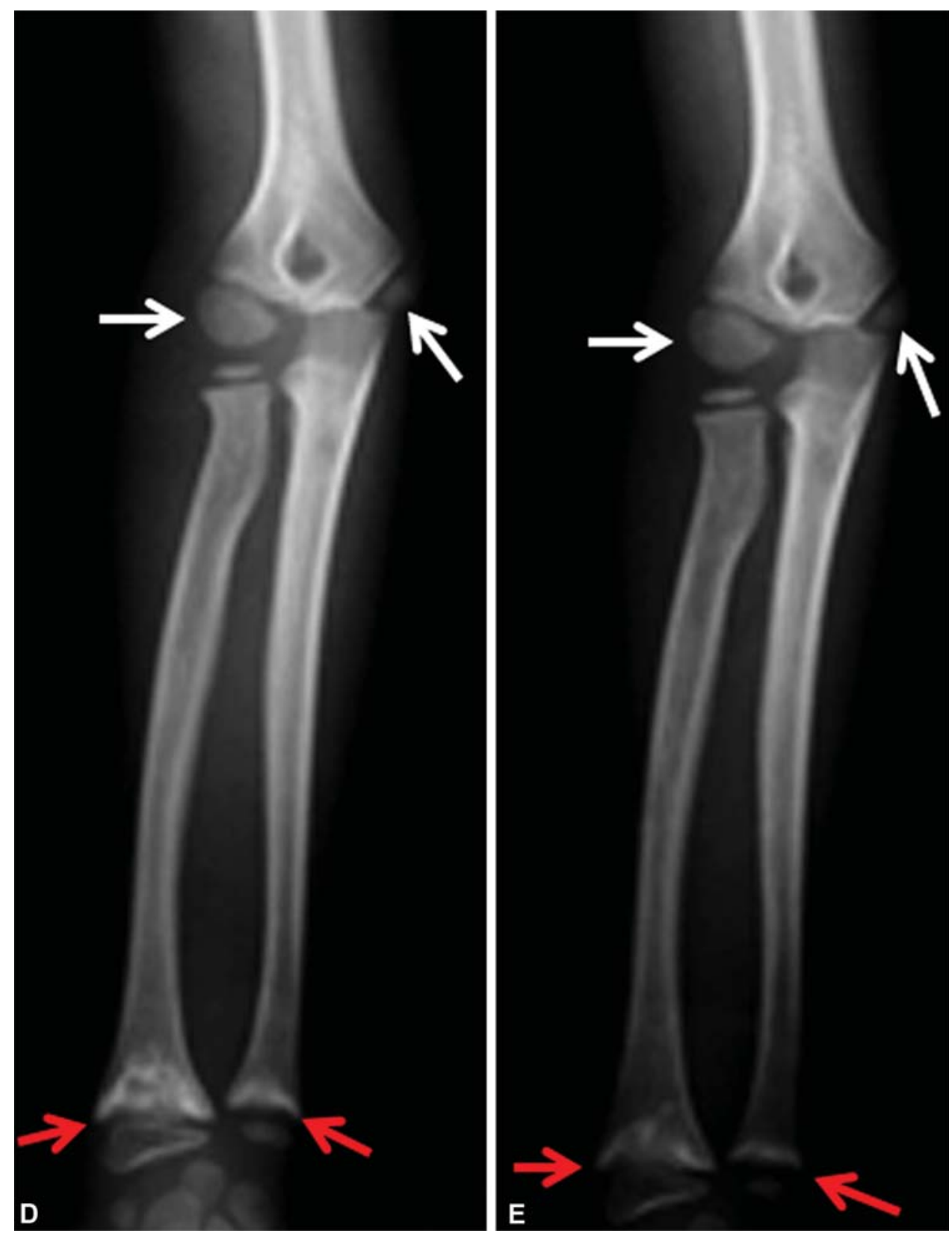

Fig. 3 (Continued)

La talla baja también es un hallazgo frecuente en la SPENCD. En casos graves, algunos pacientes tienen miembros desproporcionadamente cortos. En otros, las proporciones y la longitud del cuerpo se encuentran en el límite inferior del rango de normalidad. Se han descrito otras manifestaciones óseas, que incluyen la lordosis lumbar, la cifoescoliosis, tórax en tonel, genu valgo o genu varo.,

La SPENCD es una displasia ósea autosómica recesiva poco frecuente y heterogénea. Además de las manifestaciones óseas, se ha descrito con frecuencia compromiso neurológico, con espasticidad, retraso mental y calcificaciones cerebrales. También se han descrito alteraciones inmunológicas asociadas con ese síndrome. Sin embargo, pocas veces se ha observado inmunodeficiencia. ${ }^{3,5}$ La desregulación del sistema inmunitario en la SPENCD abarca enfermedades autoinmunes como el LES, la trombocitopenia autoinmune y la anemia hemolítica autoinmune. ${ }^{7}$ Sin embargo, sus manifestaciones clínicas y gravedad pueden ser muy variables. ${ }^{8}$

La SPENCD es causada por mutaciones bialélicas en el gen ACP5, que codifica la fosfatasa ácida resistente al tartrato (TRAP, por su sigla en inglés). En los individuos afectados se observa ausencia de expresión sérica de TRAP y, en consonancia con las manifestaciones autoinmunes, un aumento de los niveles séricos de interferón alfa (IFN $\alpha$ ) y un incremento de la expresión de los genes estimulados por interferón (ISG, por su sigla en inglés). ${ }^{1,9}$

El caso descrito destaca los principales hallazgos radiológicos observados en la SPENCD, una displasia ósea de tronco corto, caracterizada por manifestaciones 
pleiotrópicas que afectan, no solo el hueso sino también el cerebro y el sistema inmunitario.

\section{Confidencialidad de Los Datos}

Los autores declaran que han seguido los protocolos de su centro de trabajo sobre la publicación de datos de pacientes y que todos los pacientes incluidos en el estudio han recibido información suficiente y han dado su consentimiento informado por escrito.

Conflicto de Intereses

Los autores del trabajo declaran no tener ningún conflicto de intereses.

\section{Bibliografía}

1 Lausch E, Janecke A, Bros M, Trojandt S, Alanay Y, De Laet C, et al. Genetic deficiency of tartrate-resistant acid phosphatase associated with skeletal dysplasia, cerebral calcifications and autoimmunity. Nat Genet. 2011;43(02):132-137

2 Online Mendelian Inheritance in Man. OMIM (TM). McKusickNathans Institute of Genetic Medicine, Johns Hopkins University (Baltimore, MD) and National Center for Biotechnology Information, National Library of Medicine (Bethesda, MD), 2007. World Wide Web URL: http://www.ncbi.nlm.nih.gov/omim/
3 Renella R, Schaefer E, LeMerrer M, Alanay Y, Kandemir N, Eich G, Costa $\mathrm{T}$, et al. Spondyloenchondrodysplasia with spasticity, cerebral calcifications, and immune dysregulation: clinical and radiographic delineation of a pleiotropic disorder. Am J Med Genet A. 2006;140A(06):541-550

4 Schorr S, Legum C, Ochshorn M. Spondyloenchondrodysplasia. Enchondromatomosis with severe platyspondyly in two brothers. Radiology. 1976;118(01):133-139

5 Navarro V, Scott C, Briggs TA, Barete S, Frances C, Lebon P, et al. Two further cases of spondyloenchondrodysplasia (SPENCD) with immune dysregulation. Am J Med Genet A. 2008;146A(21): 2810-2815

6 Menger H, Kruse K, Spranger J. Spondyloenchondrodysplasia. J Med Genet. 1989;26(02):93-99

7 Girschick H, Wolf C, Morbach H, Hertzberg C, Lee-Kirsch MA. Severe immune dysregulation with neurological impairment and minor bone changes in a child with spondyloenchondrodysplasia due to two novel mutations in the ACP5 gene. Pediatr Rheumatol Online J. 2015;13(01):37

8 Briggs TA, Rice GI, Adib N, Ades L, Barete S, Baskar K, et al. Spondyloenchondrodysplasia due to mutations in ACP5: a comprehensive survey. J Clin Immunol. 2016;36(03): 220-234

9 Briggs TA, Rice GI, Daly S, Urquhart J, Gornall H, Bader-Meunier B, et al. Tartrate-resistant acid phosphatase deficiency causes a bone dysplasia with autoimmunity and a type I interferon expression signature. Nat Genet. 2011;43(02):127-131 